\title{
Correction to: Projection from the Anterior Cingulate Cortex to the Lateral Part of Mediodorsal Thalamus Modulates Vicarious Freezing Behavior
}

\author{
Chaowen Zheng ${ }^{1,2} \cdot$ Yanwang Huang $^{1,2} \cdot$ Binshi Bo $^{1} \cdot$ Lei Wei $^{1} \cdot$ Zhifeng Liang $^{1} \cdot$ \\ Zuoren Wang ${ }^{1,2,3}$
}

Published online: 28 August 2020

(C) Shanghai Institutes for Biological Sciences, CAS 2020

\section{Correction to: Neurosci. Bull. https://doi.org/10.1007/s12264-019-00427-z}

The original version of this article unfortunately contained some mistakes.
The authors found that in this article, the left panel of Figure 6B was a wrong placed figure (it was duplicated with the left panel of Figure 5A), and the corrected Figure 6 was shown as follow.

The original article can be found online at https:// doi.org/10.1007/s12264-019-00427-z.

\section{Zuoren Wang}

zuorenwang@ion.ac.cn

1 Institute of Neuroscience, State Key Laboratory of Neuroscience, CAS Center for Excellence in Brain Science and Intelligence Technology, Chinese Academy of Sciences, Shanghai 200031, China

2 University of Chinese Academy of Sciences, Beijing 100049, China

3 School of Future Technology, University of Chinese Academy of Sciences, Beijing 100049, China 


\section{A}

AAV-hSyn-hChR2-EGFP-ER2-WPRE-PolyA
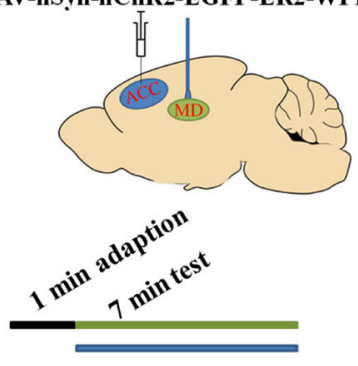

Blue light $(473 \mathrm{~nm})$

Stimulation $(5 \mathrm{~mW}, 20 \mathrm{~Hz})$

\section{B}
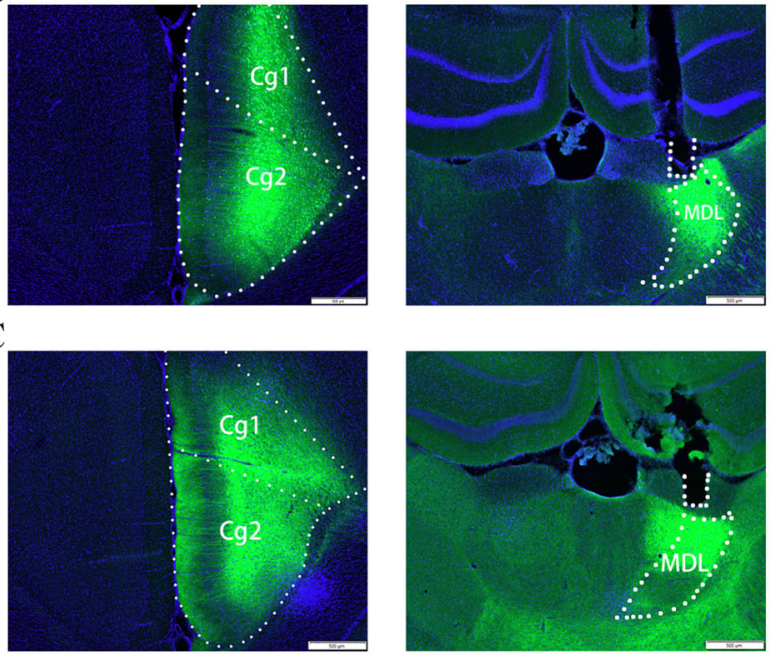

D

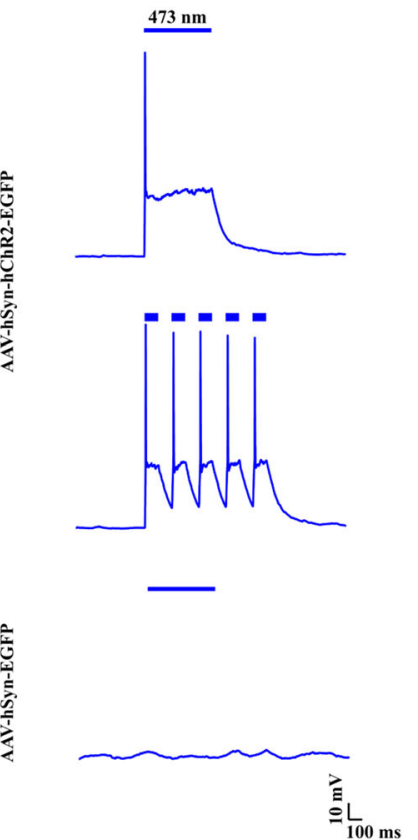

$\mathrm{E}$

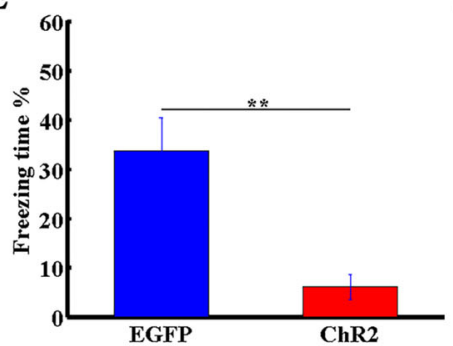

F

G
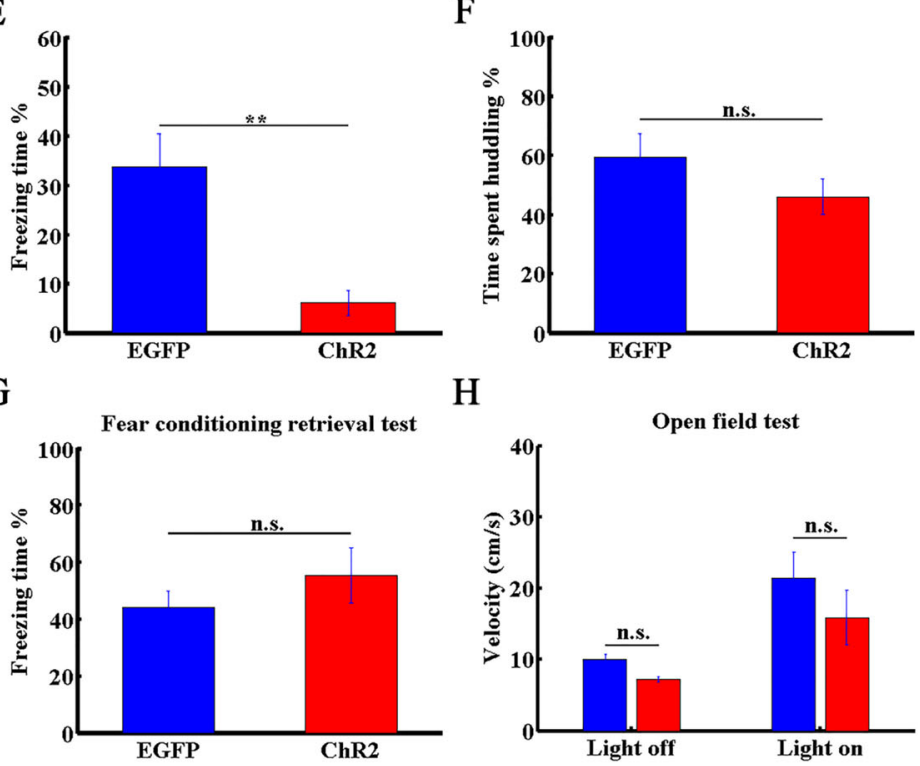

$\mathrm{H}$

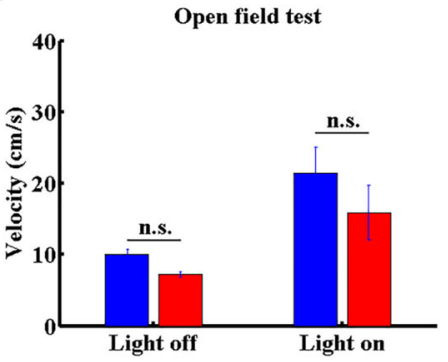

Fig. 6 Activation of the ACC-to-MDL projection decreases vicarious freezing behavior. A Diagram showing optogenetic manipulation. B Tracing of projection from the ACC to the MDL. Left panel, AAVhSyn-EGFP virus injection target in the ACC. $\mathrm{Cg} 1,2$, cingulate cortex 1 and 2; right panel, projection terminals and optical fiber position in the MDL. C Left panel, AAV-hSyn-hChR2-EGFP virus injection target in the ACC; right panel, projection terminals and optical fiber position in the MDL. Nuclei stained with DAPI. D Light pulse-induced response of ChR2-expressing (upper and middle, 10
$\mathrm{mW}, 1 \mathrm{~Hz}$ and $5 \mathrm{~Hz}$, respectively) and EGFP-expressing (lower, 10 $\mathrm{mW}, 1 \mathrm{~Hz})$ ACC neurons in brain slice. E, F Freezing (E) and huddling (F) times of OSs in AAV-hSyn-EGFP (blue) or AAV-hSynChR2 (red) groups. G Percentage freezing time of OSs in AAV-hSynEGFP- and AAV-hSyn-ChR2-injected groups in the retrieval period after fear conditioning. H Locomotor velocity of OSs in AAV-hSynEGFP (blue) and AAV-hSyn-ChR2 (red) groups, before and after light stimulation. Data are presented as the mean $\pm \mathrm{SEM}$; $* * P<0.01$, non-parametric rank-sum test, n.s., not significant. 